\title{
Epigenetic alterations in pediatric sleep apnea
}

\author{
Emily C. Cheung ${ }^{1}$, Matthew W. Kay ${ }^{1}$, Kathryn Jaques Schunke ${ }^{1,2}$
}

${ }^{1}$ Department of Biomedical Engineering, George Washington University, 800 22nd Street NW, Washington, DC 20052, USA

${ }^{2}$ Department of Anatomy, Biochemistry \& Physiology, Johns A. Burns School of Medicine, University of Hawaii, 651 Ilalo Street, Honolulu, HI 96813, USA

\section{Addresses for Correspondence}

Kathryn Jaques Schunke, PhD

Assistant Professor of Anatomy, Biochemistry \& Physiology

John A. Burns School of Medicine

University of Hawaii

651 Ilalo Street, Honolulu, HI 96813

Tel: 808-692-1565

Email: kschunke@hawaii.edu

\begin{abstract}
Pediatric obstructive sleep apnea (OSA) has significant negative effects on health and behavior in childhood including depression, failure to thrive, neurocognitive impairment, and behavioral issues. It is strongly associated with an increased risk for chronic adult disease such as obesity and diabetes, accelerated atherosclerosis, and endothelial dysfunction. Accumulating evidence suggests that adult-onset non-communicable diseases may originate from early life through a process by which an insult applied at a critical developmental window causes long-term effects on the structure or function of an organism. Recently, much attention has been paid to the role of epigenetic mechanisms in the pathogenesis of adult disease susceptibility. Epigenetic mechanisms that influence adaptive variability include histone modifications, non-coding RNAs, and DNA methylation. This review will highlight what is currently known about the phenotypic associations of epigenetic modifications in pediatric OSA and will emphasize the importance of epigenetic changes as both modulators of chronic disease and potential therapeutic targets.
\end{abstract}

\section{Introduction}

Pediatric obstructive sleep apnea (OSA) is a disorder characterized by recurring upper airway obstruction leading to intermittent oxygen desaturations, sustained levels of carbon dioxide, frequent waking during the night, habitual snoring, and excessive daytime sleepiness in children. It is estimated to affect as many as $13 \%$ of children between the ages of 3-6 years old, and $2-4 \%$ of middle-school age children ${ }^{1,2}$. Infants are particularly vulnerable to obstructive sleepdisordered breathing due to multiple predisposing factors that are often associated with infants, including their small upper airway structure, immature pulmonary mechanics and ventilatory control, high arousal threshold, insensitive laryngeal chemoreflex, and a REM-predominant sleep state $^{3}$. Childhood obesity is also a significant risk factor for OSA ${ }^{4}$ and reciprocally, OSA can contribute to obesity ${ }^{5}$. With almost 170 million children currently considered obese, and at least half of those children maintaining obesity into adulthood ${ }^{6}$, the number of children with undiagnosed sleep apnea is likely very high. The risk of pediatric OSA also increases in vulnerable populations, such as children from low socioeconomic backgrounds, especially preterm infants ${ }^{7}$. 
For example, one study found that infants born prior to 32 weeks' gestation, despite having normal birth weights, had a higher likelihood of OSA diagnosis later in childhood ${ }^{8}$.

Untreated pediatric OSA often leads to developmental deficits, such as cognitive impairments, attention-deficit/hyperactivity disorder, and poor academic performance ${ }^{9}$. Pediatric OSA is also associated with cardiovascular dysfunction and deleterious changes in metabolism. In addition to obesity ${ }^{4}$ and increased insulin resistance ${ }^{10}$, cardiovascular dysfunction includes elevated blood pressure ${ }^{11}$, increased systemic proinflammatory cytokines ${ }^{12-14}$, cardiac left ventricular hypertrophy ${ }^{15}$, and endothelial dysfunction ${ }^{16}$. These vascular, metabolic, and cognitive manifestations of OSA during a child's developmental period can have major consequences later in life and are associated with chronic adult disease such as hypertension ${ }^{11}$, diabetes mellitus ${ }^{17,18}$, atherosclerosis, and myocardial infarction ${ }^{19}$. These outcomes from more than a decade of research underscore that pediatric OSA is a major risk factor for severe health consequences in adulthood.

Clinical and experimental evidence suggest that a hallmark of OSA, chronic intermittent hypoxia $(\mathrm{CIH})$, is a major contributor to the deleterious consequences of OSA. Cyclical exposure to hypoxia stimulates sympathetic nerve activity, generates reactive oxidative species (ROS), and stabilizes hypoxia inducible factor 1 (HIF-1) (reviewed in ${ }^{20}$ ). HIF is a transcription factor involved in oxygen homeostasis and the regulation of various adaptive responses to hypoxia, including angiogenesis, metabolic reprogramming, and cell survival ${ }^{21,22}$. Animal models of OSA have shown that endothelial dysfunction, vascular remodeling, systemic and pulmonary arterial hypertension, and heart failure can develop in response to chronic intermittent hypoxia $(\mathrm{CIH})^{23-28}$ and that HIF1 stabilization is critical for eliciting many of these responses ${ }^{29}$. Many of the hypoxia-mediated perturbations of gene expression by epigenetic mechanisms are attributed to these OSA comorbidities. Such heritable, but reversible, epigenetic alterations, including DNA methylation, histone modifications, and the activation of long- and short- non-coding RNAs (IncRNA \& miRNA, respectively), have been observed in response to hypoxia and $\mathrm{ClH}{ }^{30-33}$. Importantly, drugs targeting the enzymes that catalyze these dynamic modifications are used for clinical management of some cancers ${ }^{34}$ and are in clinical trials for neurological disorders and cardiovascular diseases (reviewed $\mathrm{in}^{35}$ ). Such drugs, or drugs having similar targets, may also benefit pediatric OSA patients by lowering their risk of chronic adult disease; however, there is limited information about the associations between pediatric OSA and epigenetic alterations. A goal of this review is to highlight what is currently known about those associations and to emphasize the importance of epigenetic changes as both modulators of chronic disease and potential druggable therapeutic targets.

\section{Current Treatments for Pediatric OSA}

The most common treatment for OSA in children is adenoidectomy and tonsillectomy $(A \& T)$. A\&T has highly variable efficacy, and results have shown that the effects of the surgery may only be temporary ${ }^{36-38}$. Continuous positive airway pressure, or CPAP, which is widely used to treat adult OSA, improves vigilance and cognitive function, reduces insulin resistance, and is moderately effective in lowering blood pressure in adults with OSA and refractory hypertension. However, the use of CPAP was not found to be associated with reduced risks of cardiovascular disease, diabetes mellitus, or death for patients with OSA in recent randomized clinical trials ${ }^{39-43}$. Additionally, adherence to CPAP can be difficult for adults, and even more so in children, especially those with behavioral problems and developmental delays ${ }^{44}$. For children with mild OSA (apnea-hypopnea index (AHI) between 1 and 5), high-potency corticosteroids applied intranasally has led to mild improvements in $\mathrm{AHI}$ and blood oxygen levels ${ }^{45}$. These steroids can reduce the secretion of inflammatory cytokines IL-6, IL-8, and TNF- $\alpha^{9}$. Nevertheless, as little is known about the longitudinal effects of pediatric OSA and whether these current treatments for varying levels of severity will prevent long-term consequences of hypoxia, there is a need for more targeted therapeutic treatments. 


\section{Epigenetic mechanisms in OSA}

The epigenome harbors important clues regarding the molecular mechanisms underlying human adaptation or maladaptation to stimuli ${ }^{46-48}$. Epigenetic mechanisms that modulate gene regulation include DNA methylation, histone post translational modifications, and noncoding RNAs, such as microRNAs and long noncoding RNAs. All of these gene regulators are influenced by the environment and are likely to have an important role in the pediatric basis of adult disease susceptibility ${ }^{49}$. These epigenetic modifications alter both the chromatin organization and accessibility of genes for transcription factor binding as well as the rates of gene transcription. Chromatin organization is intimately linked to varied states experienced by any cell in its lifetime, whereby many chromatin changes occur during development in mammals ${ }^{50,51}$, in embryonic stem cells transitioning to a differentiated state in vitro ${ }^{52}$, and in various diseases ${ }^{53}$. For example, epigenetic alterations have been associated with hypoxia in cancer and a highly complex hypoxiaepigenetic interaction is observed during carcinogenesis and tumor progression ${ }^{54}$. Given the importance of epigenetics in influencing cell functions, a better understanding of both normal and abnormal epigenetic processes will provide deeper insight into the development of pediatric OSA and may reveal potential therapies involving epigenetic mechanisms. The following sections briefly explain the three primary epigenetic regulatory mechanisms (DNA methylation, histone modifications, and noncoding RNAs) and discuss their impact on long-term health during pediatric OSA.

\section{DNA methylation}

During the past decade, investigations of epigenetic modification in OSA have become more frequent and largely focus on DNA methylation in circulating leukocytes. DNA methylation is a heritable epigenetic mark involving the covalent transfer of a methyl group to the C-5 position of the cytosine ring of DNA. The mechanism by which DNA methylation regulates gene expression involves blocking the binding of transcription factors to DNA and the recruitment of proteins containing a methylated CpG-binding domain to inhibit gene expression. DNA methylation is catalyzed by DNA methyltransferases (DMNTs). DNMT1 is generally responsible for maintenance of methylation, while DNMT3a and DNMT3b perform de novo methylation ${ }^{55}$. Depending on the DNA sequence methylated, hypermethylation and hypomethylation can have either an activating or suppressive effect on gene expression, although hypermethylation is generally considered repressive.

Although there are few clinical studies of methylation patterns in pediatric OSA patients, several have identified important patterns that are relevant for inflammation and disease. One study examined genomic DNA extracted from blood samples of pediatric OSA patients. The investigators found reduced expression and hypermethylation at intron1 of Forkhead Box P3 $(\text { FOXP3 })^{56}$, a gene which is involved in T-lymphocyte differentiation and development as well as the regulation of T-cells and balancing of cytokines ${ }^{56}$. Methylation of the FOXP3 gene was directly correlated with OSA severity, as measured by apnea hypopnea index (AHI). This led to the postulation that during pediatric OSA, FOXP3 methylation suppresses FOXP3 gene expression, causing an imbalance of the Th1/Th2 cytokines. Conversely, a recent study of the development of subclinical atherosclerosis in adult patients with OSA found that plasma FOXP3 gene expression was no different than that of control patients ${ }^{57}$, demonstrating the probable and important role of developmental maturation in disease manifestation and progression. Another study investigating endothelial dysfunction in pediatric OSA patients found hypermethylation of endothelial nitric oxide synthase (eNOS) at the core promoter region of the gene with concomitant reduction of eNOS activity and associated peripheral vascular dysfunction ${ }^{16}$.

Using $\mathrm{CIH}$ as a preclinical model to phenocopy OSA, Nanduri et al demonstrated that adult rats that were exposed to $\mathrm{ClH}$ as neonates exhibited exaggerated responses to hypoxia that originated from the carotid body, the organ that regulates cardio-respiratory function. Neonatal 
$\mathrm{CIH}$ resulted in carotid body hypoxic hypersensitivity that caused irregular breathing, apnea, and hypertension in the adult rats ${ }^{58}$. Enhanced carotid body hypoxic sensitivity was associated with elevated oxidative stress, decreased expression of genes encoding antioxidant enzymes, and increased pro-oxidant enzymes. Decreased expression of the Sod2 gene in the carotid body, which encodes the antioxidant enzyme superoxide dismutase 2, was associated with DNA hypermethylation of a $\mathrm{CpG}$ dinucleotide close to the transcription start site of that gene. Importantly, treating neonatal rats with decitabine, an inhibitor of DNA methylation, during $\mathrm{ClH}$ exposure prevented the oxidative stress, enhanced carotid body hypoxic sensitivity, and reduced autonomic dysfunction that was observed in untreated neonatal rats ${ }^{58}$. These findings underscore a role for epigenetic regulation of the genome in mediating neonatal programming of carotid body hypoxic sensitivity that is maintained into adulthood, and present evidence for targeted therapy of epigenetic marks for re-programming.

\section{Histone Modifications}

The study of histone post translational modifications (PTMs) during sleep apnea is an emerging field. Understanding the global effects of individual histone modifications that culminate to create a "histone code" has been an important goal for determining the role these PTMs have in modulating chromatin accessibility. In response to periods of hypoxia during OSA, local histone PTMs can function to either activate or suppress the transcription of genes that ultimately results in a sustained response to the hypoxic periods. Although histone modifications are imposed by phosphorylation, methylation, acetylation, ubiquitylation, and sumoylation of various amino acid residues $^{59}$, methylation and acetylation of lysine residues are the most studied in OSA.

Histone methylation is the addition of methyl groups to histone tail residues. Methylation of histone tails is governed by positive and negative regulators, and each mark can have an activating or suppressive effect on transcription depending on the amino acid residue ${ }^{60}$. Achieved through the action of histone methyltransferases or histone demethylases, histone lysine methylation can occur in three different states: mono-, di- or trimethylation ${ }^{60}$. Typically, the marks that activate gene transcription include the di- or trimethylation at the H3K4, H3K36, and H3K79 sites $^{61,62,63}$. While H3K4 trimethylation acts on enhancer and promoter regions, H3K36 and H3K79 act over gene bodies ${ }^{61,62,63}$. H3K9 and H3K27 methylations are typically considered repressive ${ }^{64}$. While our understanding of activating or repressive histone methylation marks has grown over the past decades, specific methylation marks associated with pediatric sleep apnea have yet to be elucidated. Accumulating evidence from adult OSA studies confirm the presence and importance of these marks in response to $\mathrm{CIH}$. In a recent study of adult mice exposed to $\mathrm{CIH}$, macrophages isolated from the aorta had significant accumulation of the repressive histone mark H3K27me3 associated with anti-inflammatory and glutathione redox pathway member genes that protect against atherosclerosis, such as peroxisome proliferator-activated receptor/retinoid $X$ receptor and liver $X$ receptor/retinoid $X$ receptor $^{65}$. These epigenetic changes occurred in parallel with recruitment of macrophages to the aortic wall and the triggering of atherogenesis. These results indicate that histone modification-mediated activation of the oxidative stress and inflammatory pathways may be involved in the establishment of $\mathrm{ClH}$-induced endothelial dysfunction, atherosclerosis, and aortic remodeling in OSA. More studies of pediatric OSA are needed to establish the plasticity of these marks from childhood into adulthood and the associated disease progression.

Histone acetylation occurs when an acetyl group is transferred onto the lysine residue of a histone tail and generally contributes to increased accessibility of transcriptional machinery to chromatin. Histone acetylation is achieved through the catalytic enzymes known as histone acetyltransferases (HATs). Conversely, histone deacetylases (HDACs) function to decrease histone acetylation, thereby reducing accessibility of chromatin to transcriptional regulators. HDACs are stratified into four different classes based on their structure and function ${ }^{66}$. HDAC expression and subsequent histone acetylation patterns have been studied minimally in adult 
OSA, and results suggest a direct correlation with disease phenotype. However, there are no published reports on this mechanism in pediatric OSA. We will next briefly discuss the current literature on histone acetylation to highlight the importance of these modifications in adult disease.

In adult patients with moderately severe OSA, a Class III HDAC (SIRT1) is reduced in peripheral blood cells. SIRT1 has an important regulatory role over transcriptional regulators such as p53, NF-kB, eNOS, and FOXO. Moreover, disruption of SIRT function has been implicated in metabolic and cardiovascular disease. In OSA adult patients, expression and activity of SIRT1, as well as plasma NOX, were restored following 3 months of CPAP ${ }^{67}$. This result highlights both the potential plasticity of inducing and removing histone acetylation and the significant role of acetylation in pathogenesis. In a separate study, a number of gene sets were found to be upregulated in the adipose tissue of OSA patients, including genes of the pro-inflammatory NF$\mathrm{kB}$ pathway and the proteolytic ubiquitin/proteasome module ${ }^{68}$. Further network analysis of these pathways suggested HDAC2 as a hub protein for this dysregulation ${ }^{69}$. Indeed, HDAC2 has previously been shown to physically interact with FOXO3a and regulate FOXO3a-dependent gene transcription during oxidative stress ${ }^{70}$. In a preclinical study, macrophages isolated from the aorta of adult mice exposed to $\mathrm{ClH}$ had significant accumulation of the active histone mark $\mathrm{H} 3 \mathrm{~K} 9 \mathrm{Ac}$ associated with pro-inflammatory and oxidative stress signaling pathway members, including HIF-1, p53, NF-kB, tumor growth factor- $\beta$, FOXO4, and IL-6 ${ }^{65}$. Other common acetylation marks that are activated at enhancer and promoter regions include $\mathrm{H} 3 \mathrm{~K} 27 \mathrm{Ac}^{71}$ and $\mathrm{H} 4 \mathrm{~K} 16 \mathrm{Ac}^{72}$; however, there is a general lack of knowledge regarding how these marks contribute to adult or pediatric obstructive sleep apnea.

Even though histone marks are endogenously reversible, histone modifications that develop in childhood due to sleep apnea are likely to have a long-lasting effect on the cardiovascular system that will continue with the child into adulthood, as is evidenced by studies of DNA methylation ${ }^{58}$. The plasticity of chromatin regulation makes targeting the enzymatic machinery, or the reversible alterations themselves, an attractive strategy for therapeutic intervention. In fact, an increasing number of small molecule inhibitors designed to counteract a variety of epigenetic regulators are currently under development or are already used clinically. Epigenetic biomarkers and targeted epigenetic therapies such as the histone deacetylase (HDAC) inhibitors Vorinostat, Panobinostat, Romidepsin and Belinostat, are approved for certain lymphomas and myeloma. Cardiovascular studies using HDAC inhibitors have shown promise in pre-clinical management of cardiac hypertrophy, heart failure, oxidative stress, hypertension and cardiac fibrosis $^{73-76}$. Recently, Givinostat, a clinical-stage inhibitor of HDAC catalytic activity was shown efficacious in two distinct murine models of diastolic dysfunction with preserved ejection fraction, by relieving impaired cardiac myofibril relaxation ${ }^{77}$. HDAC inhibitors have also been shown to have a dose-dependent anti-inflammatory effect, decreasing inflammatory cytokines such as TNF- $\alpha$ and IFN- $\beta$ in models of irritable bowel disease ${ }^{78}$. Determining the extent of epigenetic alterations that occur in response to pediatric OSA and the mechanisms by which they influence the neonatal basis of disease susceptibility and progression is required for development of new epigenetic targeted treatments for OSA in neonates and adults.

\section{Noncoding RNAs}

Noncoding RNAs consist of microRNAs (miRNAs) and long non-coding RNAs (IncRNAs). Over the past decade, the understanding of the role of RNA has shifted; there is increasing evidence that only $1-2 \%$ of RNA codes for proteins ${ }^{79,80}$, while non-coding RNA has a predominant and essential role in modulating gene expression. MicroRNAs are single-stranded RNAs that are 18-22 nucleotides in length and negatively regulate gene expression at the post transcriptional level by binding to mRNA, which can then lead to translational repression ${ }^{81,82}$. Considered an ideal biomarker in the era of precision medicine, miRNAs have the capacity to regulate most protein encoding genes; thus, the up or down-regulation of certain miRNAs can have downstream effects on target genes. 
MicroRNAs have been studied extensively in adult OSA, but comparatively little is known about miRNAs in pediatric OSA. One study, using peripheral blood serum samples from adult OSA patients, showed differential expression of multiple microRNAs, including upregulation of miR-574-5p and miR-139-3p, and downregulation of miR-485-5p, miR-107, miR-574-5p, and miR-199-3 ${ }^{83}$. Target genes for these miRNAs include CYP21A2, CAD, PANK, and others, which have a key role in the metabolism of lipids, along with insulin, PI3K-AKT, and mTOR signaling pathways ${ }^{83}$. Overall, these miRNAs have been shown to be involved in regulation of metabolism, hypoxia, and oxidative stress. A more recent study evaluating miRNAs present in plasma of OSA patients indicated decreased circulating concentrations of miR-181a, miR-199b, miR-345, miR133a, miR-340 and miR-486-3p ${ }^{82}$. Of these miRNAs, miR-181a and miR-345 levels correlated with the apnea hypopnea index $(\mathrm{AHI})^{82}$. In gestational hypoxia, one microRNA that has been studied extensively is miR-210. Elevated levels of circulating placental miR-210 has been demonstrated in gestational hypoxia, high altitude pregnancies, and preclampsia ${ }^{84-87}$.

To date, only two clinical studies have investigated miRNAs in children with OSA. Downregulation of miR-630 was observed in plasma exosomes of children aged 4-12 with OSA and was linked to endothelial dysfunction, an early risk factor for atherosclerosis and cardiovascular disease ${ }^{88}$. Importantly, exogenous miR-630 administered to human endothelial cells in vitro restored endothelial function. Another study found upregulation of miR-92a in the serum of both adults and children diagnosed with OSA $^{89}$. The family of miR-92a has also been identified as a promoter of tumor proliferation in several human cancers ${ }^{90}$ and may serve as a useful biomarker of OSA and potential risk marker for cardiovascular disease.

Long non-coding RNAs consist of RNA strands that are longer than 200 nucleotides $^{91}$. These IncRNAs can be up- or downregulated in disease progression and can modulate gene expression through a variety of mechanisms such as altering chromatin recruitment or chromatin modifiers, and can suppress transcription initiation by interfering with RNA polymerase ${ }^{91}$. LncRNAs have been studied in the development of obesity, with implications in the process of adipogenesis through the modulation of the gene expression of cell cycle marker genes such as cyclin $B, D$, and $E^{92}$. LncRNAs also have a key function in mitigating myocardial infarction and myocardial ischemia-reperfusion injury, and heart failure (reviewed in ${ }^{93}$ ). There are numerous publications that describe the role of IncRNAs in metabolic syndromes such as obesity, type II diabetes, cardiovascular disease, and pulmonary hypertension, all of which are comorbidities of sleep apnea. However, only a few published studies have investigated the role of long noncoding RNAs in the genesis and progression of OSA in adults and more importantly, in children.

In an adult rat model of OSA, decreased expression of IncRNA CPS1-intronic transcript was linked to pulmonary artery hypertension, and exogenous overexpression of IncRNA CPS1IT alleviated hypertension through decreased IL-1 $\beta$ expression via inhibition of HIF-1 transcriptional activity and the NF-k $\beta$ signaling pathway ${ }^{94}$. A recent study by Zhou et $\mathrm{al}^{95}$ found that IncRNA XIST promotes inflammation through the reduction of glucocorticoid receptor alpha in the adenoids of children with OSA. To our knowledge, this is the only published report to-date of IncRNA expression profiles in children with OSA, despite the abundance of compelling evidence that IncRNA are differentially regulated in response to cyclic hypoxia and are linked to cardiovascular disease. A deeper understanding of how IncRNAs regulate gene expression in pediatric sleep apnea will likely identify promising new therapeutic targets that could prevent the severe adult complications and disease that originate from childhood OSA.

\section{Concluding remarks}

Pediatric OSA has significant negative effects on behavior and health in children, including depression, failure to thrive, neurocognitive impairment, excessive daytime sleepiness, increased risk for systemic and pulmonary hypertension, and behavioral issues suggestive of attentiondeficit/hyperactivity disorder ${ }^{96,97}$. Importantly, pediatric OSA is strongly associated with an increased risk for a variety of end-organ injury and dysfunction in adults, such as obesity and 
diabetes, accelerated atherosclerosis, and endothelial dysfunction; syndromes that impose both immediate and long-term morbidities resulting in high healthcare costs ${ }^{3,37}$. Deeper investigations of epigenetic regulators that interact to modify gene expression will provide not only detailed mechanistic understanding of disease progression but also new insights into the fetal basis of adult disease susceptibility. Building upon the evidence that DNA methylation, post-translational histone modifications, and noncoding RNAs all modulate cellular reprogramming due to exposure to hypoxia, a thorough understanding of how these chromatin modifications work synergistically will be crucial for developing the most effective targeted treatments. The promise is that such treatments will target the most fundamental underpinnings of chronic disease by blunting the deleterious epigenetic programming that occurs during pediatric OSA. Gaining a broader understanding of how epigenetic regulators are modulated during a child's developmental period and are dysregulated due to cyclic hypoxia is an extremely important direction for the future of pediatric OSA research.

\section{Author Contributions}

All authors have read and agreed to the published version of the manuscript. Writing - original draft preparation, E.C.; review and editing, M.W.K., K.J.S.; funding acquisition, K.J.S, M.W.K.

\section{Funding}

This publication was supported by UL1TR001876 from the NIH NCATS to K.J.S, and by NIH NHLBI R01s HL146169 \& HL144157 to M.W.K. Its contents are solely the responsibility of the authors and do not necessarily represent the official views of the National Center for Advancing Translational Sciences or the National Institutes of Health.

\section{Conflicts of Interest}

The authors declare no conflict of interest. The funders had no role in the writing of the manuscript or in the decision to publish.

\section{References}

1. levers-Landis, C. E. \& Redline, S. Pulmonary Perspective Pediatric Sleep Apnea Implications of the Epidemic of Childhood Overweight. Am J Respir Crit Care Med 175, (2007).

2. Bixler, E. O. et al. Sleep disordered breathing in children in a general population sample: Prevalence and risk factors. Sleep 32, 731-736 (2009).

3. Katz, E. S., Mitchell, R. B. \& D'Ambrosio, C. M. Obstructive sleep apnea in infants. Am. J. Respir. Crit. Care Med. 185, 805-16 (2012).

4. Verhulst, S. L., Van Gaal, L., De Backer, W. \& Desager, K. The prevalence, anatomical correlates and treatment of sleep-disordered breathing in obese children and adolescents. Sleep Med. Rev. 12, 339-346 (2008).

5. Wolk, R., Shamsuzzaman, A. S. M. \& Somers, V. K. Obesity, Sleep Apnea, and Hypertension. Hypertension 42, 1067-1074 (2003).

6. Motevalli, M., Drenowatz, C., Tanous, D. R., Khan, N. A. \& Wirnitzer, K. Management of childhood obesity - time to shift from generalized to personalized intervention strategies. Nutrients 13, (2021).

7. Calhoun, S. L. et al. Prenatal and perinatal complications: Is it the link between race and SES and childhood sleep disordered breathing? J. Clin. Sleep Med. 6, 264-269 (2010).

8. Raynes-Greenow, C. H. et al. Sleep apnea in early childhood associated with preterm birth but not small for gestational age: A population-based record linkage study. Sleep 35, 1475-1480 (2012).

9. Tan, H. L., Gozal, D. \& Kheirandish-Gozal, L. Obstructive sleep apnea in children: A critical update. Nature and Science of Sleep 5, 109-123 (2013). 
10. Koren, D., Gozal, D., Philby, M. F., Bhattacharjee, R. \& Kheirandish-Gozal, L. Impact of obstructive sleep apnoea on insulin resistance in nonobese and obese children. Eur. Respir. J. 47, 1152-1161 (2016).

11. Nisbet, L. C. et al. Preschool children with obstructive sleep apnea: The beginnings of elevated blood pressure? Sleep 36, 1219-1226 (2013).

12. Huang, Y. S. et al. Inflammatory cytokines in pediatric obstructive sleep apnea. Med. (United States) 95, (2016).

13. Tauman, R., O'Brien, L. M. \& Gozal, D. Hypoxemia and obesity modulate plasma Creactive protein and interleukin-6 levels in sleep-disordered breathing. Sleep Breath. 11, 77-84 (2007).

14. Gozal, D. et al. Sleep measures and morning plasma TNF- $\alpha$ levels in children with sleepdisordered breathing. Sleep 33, 319-325 (2010).

15. Kaditis, A. G. et al. Obstructive sleep disordered breathing in 2- to 18-year-old children: Diagnosis and management. Eur. Respir. J. 47, 69-94 (2016).

16. Kheirandish-Gozal, L., Khalyfa, A., Gozal, D., Bhattacharjee, R. \& Wang, Y. Endothelial dysfunction in children with obstructive sleep apnea is associated with epigenetic changes in the eNOS gene. Chest 143, 971-977 (2013).

17. Waters, K. A. et al. Follow-up on metabolic markers in children treated for obstructive sleep apnea. Am. J. Respir. Crit. Care Med. 174, 455-460 (2006).

18. De La Eva, R. C., Baur, L. A., Donaghue, K. C. \& Waters, K. A. Metabolic correlates with obstructive sleep apnea in obese subjects. J. Pediatr. 140, 654-659 (2002).

19. Gozal, D. \& Kheirandish-Gozal, L. Cardiovascular morbidity in obstructive sleep apnea: Oxidative stress, inflammation, and much more. Am. J. Respir. Crit. Care Med. 177, 369375 (2008).

20. Prabhakar, N. R., Peng, Y.-J. \& Nanduri, J. Hypoxia-inducible factors and obstructive sleep apnea. J. Clin. Invest. 130, 5042-5051 (2020).

21. Wang, G. L., Jiang, B. H., Rue, E. A. \& Semenza, G. L. Hypoxia-inducible factor 1 is a basic-helix-loop-helix-PAS heterodimer regulated by cellular $\mathrm{O} 2$ tension. Proc. Natl. Acad. Sci. U. S. A. 92, 5510-4 (1995).

22. Semenza, G. L. Hypoxia-Inducible Factor 1 and Cardiovascular Disease. Annu. Rev. Physiol. 76, 39-56 (2014).

23. Jameson, $\mathrm{H}$. et al. Oxytocin neuron activation prevents hypertension that occurs with chronic intermittent hypoxia/hypercapnia in rats. Am. J. Physiol. - Hear. Circ. Physiol. 310, H1549-H1557 (2016).

24. Campen, M. J., Shimoda, L. A. \& O'Donnell, C. P. Acute and chronic cardiovascular effects of intermittent hypoxia in C57BL/6J mice. J. Appl. Physiol. 99, 2028-2035 (2005).

25. Dematteis, M. et al. Intermittent hypoxia induces early functional cardiovascular remodeling in mice. Am. J. Respir. Crit. Care Med. 177, 227-235 (2008).

26. Fletcher, E. C., Lesske, J., Qian, W., Miller, C. C. \& Unger, T. Repetitive, episodic hypoxia causes diurnal elevation of blood pressure in rats. Hypertension 19, 555-561 (1992).

27. Farré, R. et al. Rat model of chronic recurrent airway obstructions to study the sleep apnea syndrome. Sleep 30, 930-933 (2007).

28. Dergacheva, O., Dyavanapalli, J., Piñol, R. A. \& Mendelowitz, D. Chronic intermittent hypoxia and hypercapnia inhibit the hypothalamic paraventricular nucleus neurotransmission to parasympathetic cardiac neurons in the brain stem. Hypertension 64, 597-603 (2014).

29. Peng, Y.-J. et al. Heterozygous HIF-1a deficiency impairs carotid body-mediated systemic responses and reactive oxygen species generation in mice exposed to intermittent hypoxia. J. Physiol. 577, 705-716 (2006).

30. Melvin, A. \& Rocha, S. Chromatin as an oxygen sensor and active player in the hypoxia 
response. Cell. Signal. 24, 35-43 (2012).

31. Xin, J. et al. Chromatin accessibility landscape and regulatory network of high-altitude hypoxia adaptation. Nat. Commun. 11, 4928 (2020).

32. Batie, M. et al. Hypoxia induces rapid changes to histone methylation and reprograms chromatin. Science (80-. ). 363, 1222-1226 (2019).

33. Prickaerts, P. et al. Hypoxia increases genome-wide bivalent epigenetic marking by specific gain of H3K27me3. Epigenetics Chromatin 9, 46 (2016).

34. Cheng, Y. et al. Targeting epigenetic regulators for cancer therapy: mechanisms and advances in clinical trials. Signal Transduct. Target. Ther. 4, 62 (2019).

35. Ganesan, A., Arimondo, P. B., Rots, M. G., Jeronimo, C. \& Berdasco, M. The timeline of epigenetic drug discovery: from reality to dreams. Clin. Epigenetics 11, 174 (2019).

36. Gozal, D., Tan, H.-L. \& Kheirandish-Gozal, L. Treatment of Obstructive Sleep Apnea in Children: Handling the Unknown with Precision. J. Clin. Med. 9, 888 (2020).

37. Muzumdar, H. \& Arens, R. Physiological effects of obstructive sleep apnea syndrome in childhood. Respir. Physiol. Neurobiol. 188, 370-382 (2013).

38. Muzumdar, H. \& Arens, R. Diagnostic issues in pediatric obstructive sleep apnea. Proc. Am. Thorac. Soc. 5, 263-273 (2008).

39. Yu, J. et al. Association of Positive Airway Pressure With Cardiovascular Events and Death in Adults With Sleep Apnea: A Systematic Review and Meta-analysis. JAMA 318, 156-166 (2017).

40. Liu, L., Cao, Q., Guo, Z. \& Dai, Q. Continuous Positive Airway Pressure in Patients With Obstructive Sleep Apnea and Resistant Hypertension: A Meta-Analysis of Randomized Controlled Trials. J. Clin. Hypertens. 18, 153-158 (2016).

41. Iftikhar, I. H., Khan, M. F., Das, A. \& Magalang, U. J. Meta-analysis: Continuous Positive Airway Pressure Improves Insulin Resistance in Patients with Sleep Apnea without Diabetes. Ann. Am. Thorac. Soc. 10, 115-120 (2013).

42. Labarca, G., Reyes, T., Jorquera, J., Dreyse, J. \& Drake, L. CPAP in patients with obstructive sleep apnea and type 2 diabetes mellitus: Systematic review and metaanalysis. Clin. Respir. J. 12, 2361-2368 (2018).

43. da Silva Paulitsch, F. \& Zhang, L. Continuous positive airway pressure for adults with obstructive sleep apnea and cardiovascular disease: a meta-analysis of randomized trials. Sleep Med. 54, 28-34 (2019).

44. Tam, C. S., Wong, M., McBain, R., Bailey, S. \& Waters, K. A. Inflammatory measures in children with obstructive sleep apnoea. J. Paediatr. Child Health 42, 277-282 (2006).

45. Capdevila, O. S., Kheirandish-Gozal, L., Dayyat, E. \& Gozal, D. Pediatric obstructive sleep apnea: Complications, management, and long-term outcomes. Proceedings of the American Thoracic Society 5, 274-282 (2008).

46. Julian, C. G. Epigenomics and human adaptation to high altitude. J. Appl. Physiol. 123, 1362-1370 (2017).

47. Virzì, G. M., Clementi, A., Brocca, A., de Cal, M. \& Ronco, C. Epigenetics: a potential key mechanism involved in the pathogenesis of cardiorenal syndromes. J. Nephrol. 31, 333341 (2018).

48. Zoghbi, H. Y. \& Beaudet, A. L. Epigenetics and Human Disease. Cold Spring Harb. Perspect. Biol. 8, a019497 (2016).

49. Perikleous, E. et al. DNA Methylation in Pediatric Obstructive Sleep Apnea: An Overview of Preliminary Findings. Front. Pediatr. 6, 154 (2018).

50. Filipescu, D., Müller, S. \& Almouzni, G. Histone H3 variants and their chaperones during development and disease: contributing to epigenetic control. Annu. Rev. Cell Dev. Biol. 30, 615-646 (2014).

51. Atlasi, Y. \& Stunnenberg, H. G. The interplay of epigenetic marks during stem cell differentiation and development. Nat. Rev. Genet. 18, 643-658 (2017). 
52. Meshorer, E. \& Misteli, T. Chromatin in pluripotent embryonic stem cells and differentiation. Nat. Rev. Mol. Cell Biol. 7, 540-546 (2006).

53. Mirabella, A. C., Foster, B. M. \& Bartke, T. Chromatin deregulation in disease. Chromosoma 125, 75-93 (2016).

54. Camuzi, D. et al. Regulation Is in the Air: The Relationship between Hypoxia and Epigenetics in Cancer. Cells 8, 300 (2019).

55. Chen, Y. C. et al. Aberrant DNA methylation levels of the formyl peptide receptor $1 / 2 / 3$ genes are associated with obstructive sleep apnea and its clinical phenotypes. Am. J. Transl. Res. 12, 2521-2537 (2020).

56. Kim, J. et al. DNA methylation in inflammatory genes among children with obstructive sleep apnea. Am. J. Respir. Crit. Care Med. 185, 330-338 (2012).

57. Sanz-Rubio, D. et al. Forkhead box P3 methylation and expression in men with obstructive sleep apnea. Int. J. Mol. Sci. 21, (2020).

58. Nanduri, J. et al. Epigenetic regulation of hypoxic sensing disrupts cardiorespiratory homeostasis. Proc. Natl. Acad. Sci. 109, 2515-2520 (2012).

59. Histone post-translational modifications - Latest research and news | Nature.

60. Jambhekar, A., Dhall, A. \& Shi, Y. Roles and regulation of histone methylation in animal development. Nat. Rev. Mol. Cell Biol. 20, 625-641 (2019).

61. Bernstein, B. E. et al. Genomic maps and comparative analysis of histone modifications in human and mouse. Cell 120, 169-181 (2005).

62. Bernstein, B. E. et al. Methylation of histone H3 Lys 4 in coding regions of active genes. Proc. Natl. Acad. Sci. U. S. A. 99, 8695-8700 (2002).

63. Barski, A. et al. High-Resolution Profiling of Histone Methylations in the Human Genome. Cell 129, 823-837 (2007).

64. Sasidharan Nair, V. et al. DNA methylation and repressive H3K9 and H3K27 trimethylation in the promoter regions of PD-1, CTLA-4, TIM-3, LAG-3, TIGIT, and PD-L1 genes in human primary breast cancer. Clin. Epigenetics 10, 1-12 (2018).

65. Cortese, R. et al. Aorta macrophage inflammatory and epigenetic changes in a murine model of obstructive sleep apnea: Potential role of CD36. Sci. Rep. 7, 43648 (2017).

66. Wright, L. H. \& Menick, D. R. A class of their own: Exploring the nondeacetylase roles of class Ila HDACs in cardiovascular disease. Am. J. Physiol. - Hear. Circ. Physiol. 311, H199-H206 (2016).

67. Chen, W.-J. J. et al. Effect of Nasal CPAP on SIRT1 and Endothelial Function in Obstructive Sleep Apnea Syndrome. Lung 193, 1037-1045 (2015).

68. Gharib, S. A., Hayes, A. L., Rosen, M. J. \& Patel, S. R. A pathway-based analysis on the effects of obstructive sleep apnea in modulating visceral fat transcriptome. Sleep 36, 2330 (2013).

69. Chen, J. H., Yang, R., Wang, Y. P. \& Zhang, W. Expression data analysis to identify key target genes in visceral fat tissue associated with obstructive sleep apnea. Eur. Rev. Med. Pharmacol. Sci. 19, 4293-4299 (2015).

70. Peng, S. et al. HDAC2 selectively regulates FOXO3a-mediated gene transcription during oxidative stress-induced neuronal cell death. J. Neurosci. 35, 1250-1259 (2015).

71. Zhang, T., Zhang, Z., Dong, Q., Xiong, J. \& Zhu, B. Histone H3K27 acetylation is dispensable for enhancer activity in mouse embryonic stem cells. Genome Biol. 21, 1-7 (2020).

72. Taylor, G. C. A., Eskeland, R., Hekimoglu-Balkan, B., Pradeepa, M. M. \& Bickmore, W. A. H4K16 acetylation marks active genes and enhancers of embryonic stem cells, but does not alter chromatin compaction. Genome Res. 23, 2053-2065 (2013).

73. Wallner, M. et al. HDAC inhibition improves cardiopulmonary function in a feline model of diastolic dysfunction. Sci. Transl. Med. 12, (2020).

74. McKinsey, T. A. Therapeutic potential for HDAC inhibitors in the heart. Annu. Rev. 
Pharmacol. Toxicol. 52, 303-19 (2012).

75. Kee, H. J. et al. Selective inhibition of histone deacetylase 8 improves vascular hypertrophy, relaxation, and inflammation in angiotensin II hypertensive mice. Clin. Hypertens. 25, 13 (2019).

76. Ooi, J. Y. Y. et al. HDAC inhibition attenuates cardiac hypertrophy by acetylation and deacetylation of target genes. Epigenetics 10, 418-430 (2015).

77. Jeong, M. Y. et al. Histone deacetylase activity governs diastolic dysfunction through a nongenomic mechanism. Sci. Transl. Med. 10, (2018).

78. Edwards, A. J. P. \& Pender, S. L. F. Histone deacetylase inhibitors and their potential role in inflammatory bowel diseases. in Biochemical Society Transactions 39, 1092-1095 (Biochem Soc Trans, 2011).

79. Mattick, J. S. The genetic signatures of noncoding RNAs. PLoS Genet. 5, (2009).

80. Ponting, C. P., Oliver, P. L. \& Reik, W. Evolution and Functions of Long Noncoding RNAs. Cell 136, 629-641 (2009).

81. He, L. \& Hannon, G. J. MicroRNAs: Small RNAs with a big role in gene regulation. Nature Reviews Genetics 5, 522-531 (2004).

82. Santamaria-Martos, F. et al. Circulating microRNA profile as a potential biomarker for obstructive sleep apnea diagnosis. Sci. Rep. 9, 1-9 (2019).

83. Li, K., Wei, P., Qin, Y. \& Wei, Y. MicroRNA expression profiling and bioinformatics analysis of dysregulated microRNAs in obstructive sleep apnea patients. Med. (United States) 96, (2017).

84. Ducsay, C. A. et al. Gestational hypoxia and developmental plasticity. Physiol. Rev. 98, 1241-1334 (2018).

85. Colleoni, F. et al. Suppression of Mitochondrial Electron Transport Chain Function in the Hypoxic Human Placenta: A Role for miRNA-210 and Protein Synthesis Inhibition. PLoS One 8, (2013).

86. Lee, D. C. et al. MiR-210 targets iron-sulfur cluster scaffold homologue in human trophoblast cell lines: Siderosis of interstitial trophoblasts as a novel pathology of preterm preeclampsia and small-for-gestational-age pregnancies. Am. J. Pathol. 179, 590-602 (2011).

87. Hale, A. et al. An Argonaute 2 switch regulates circulating miR-210 to coordinate hypoxic adaptation across cells. Biochim. Biophys. Acta - Mol. Cell Res. 1843, 2528-2542 (2014).

88. Khalyfa, A. et al. Circulating plasma extracellular microvesicle MicroRNA cargo and endothelial dysfunction in children with obstructive sleep apnea. Am. J. Respir. Crit. Care Med. 194, 1116-1126 (2016).

89. Gongol, B. et al. Serum miR-92a is Elevated in Children and Adults with Obstructive Sleep Apnea. J. Mol. Biomark. Diagn. 11, (2020).

90. Jiang, M., Li, X., Quan, X., Li, X. \& Zhou, B. MiR-92a Family: A Novel Diagnostic Biomarker and Potential Therapeutic Target in Human Cancers. Front. Mol. Biosci. 6, 98 (2019).

91. Yao, R. W., Wang, Y. \& Chen, L. L. Cellular functions of long noncoding RNAs. Nature Cell Biology 21, 542-551 (2019).

92. Rey, F. et al. Role of long non-coding RNAs in adipogenesis: State of the art and implications in obesity and obesity-associated diseases. Obes. Rev. 22, e13203 (2021).

93. Chen, C., Tang, Y., Sun, H., Lin, X. \& Jiang, B. The roles of long noncoding RNAs in myocardial pathophysiology. Biosci. Rep. 39, (2019).

94. Zhang, Z., Li, Z., Wang, Y., Wei, L. \& Chen, H. Overexpressed long noncoding RNA CPS1-IT alleviates pulmonary arterial hypertension in obstructive sleep apnea by reducing interleukin-1 $\beta$ expression via HIF1 transcriptional activity. J. Cell. Physiol. 234, 19715-19727 (2019).

95. Zhou, Z., Ni, H., Li, Y. \& Jiang, B. LncRNA XIST promotes inflammation by 
downregulating GRa expression in the adenoids of children with OSAHS. Exp. Ther. Med. 21, 500 (2021).

96. Bonuck, K., Freeman, K., Chervin, R. D. \& Xu, L. Sleep-disordered breathing in a population-based cohort: Behavioral outcomes at 4 and 7 years. Pediatrics 129, (2012).

97. Gottlieb, D. J. et al. Symptoms of sleep-disordered breathing in 5-year-old children are associated with sleepiness and problem behaviors. Pediatrics 112, 870-877 (2003). 